Archives

4 | 1989

Varia

\title{
Une enquête sur les constructions publiques en métal dans la région Île-de-France
}

Frédéric Seitz et Louis Bergeron

\section{Q OpenEdition}

Journals

Édition électronique

URL : http://journals.openedition.org/ccrh/2907

DOI : $10.4000 /$ ccrh.2907

ISSN : $1760-7906$

Éditeur

Centre de recherches historiques - EHESS

Édition imprimée

Date de publication : 15 octobre 1989

ISSN : 0990-9141

Référence électronique

Frédéric Seitz et Louis Bergeron, « Une enquête sur les constructions publiques en métal dans la région Île-de-France », Les Cahiers du Centre de Recherches Historiques [En ligne], 4 | 1989, mis en ligne le 13 avril 2009, consulté le 19 avril 2019. URL : http://journals.openedition.org/ccrh/2907 ; DOI : $10.4000 /$ ccrh. 2907

Ce document a été généré automatiquement le 19 avril 2019

Article L.111-1 du Code de la propriété intellectuelle. 


\title{
Une enquête sur les constructions publiques en métal dans la région Île- de-France
}

\author{
Frédéric Seitz et Louis Bergeron
}

\section{NOTE DE L'AUTEUR}

Cette recherche a été engagée à la suite d'un appel d'offres lancé par le Ministère de la Recherche en 1988, auquel ont répondu, pour le Centre de recherches historiques, Louis BERGERON et Frédéric SEITZ (ce dernier est ingénieur de recherche à l'EHESS).

1 Tous les travaux de recherche entrepris au cours de ces dernières années sur l'architecture française des $\mathrm{XIX}^{\mathrm{e}}$ et $\mathrm{Xx}^{\mathrm{e}}$ siècles ont rappelé l'absence de répertoire et de dictionnaire apportant des informations claires et précises tant sur la nature des constructions réalisées durant cette période que sur les architectes et les ingénieurs qui en sont les auteurs.

2 Le Centre de recherches historiques a ainsi élaboré le projet de mettre sur pied un instrument de travail scientifique dont l'objet est de combler le vide maintes fois souligné, dans un domaine spécifique : celui de l'architecture métallique.

3 Cette architecture connait un sort particulier dans notre pays car elle est non seulement en général totalement méconnue, mais, de plus, lorsque par hasard on la remarque, c'est pour lui attribuer tous les soi-disant maux de la construction moderne et contemporaine : absence de solidité, confort médiocre, aspect déplorable, etc. Son approche reste très superficielle et aucun des problèmes fondamentaux qu'elle soulève n'a été posé. Or, l'étude de l'histoire de ce type d'architecture est très riche d'enseignements. 


\section{Une longue évolution}

4 Rappelons d'abord le caractère permanent de l'utilisation du métal dans l'architecture française moderne et contemporaine. Ce matériau est en effet présent dans l'art de bâtir depuis fort longtemps et les constructeurs n'ont pas hésité à l'utiliser dès que l'état des techniques industrielles l'a permis, cette utilisation ayant pris des aspects très différents et ayant généré des oeuvres très diverses dans le temps et dans l'espace.

5 Dans un premier temps, le fer s'introduisit, au XIX ${ }^{e}$ siècle, dans le domaine bâti sous la forme de structures inspirées des méthodes de construction antérieures. Permettant l'augmentation des portées et des surfaces couvertes et étant ainsi à l'origine des bâtiments nouveaux réclamés par une société en pleine mutation, le métal évolue ensuite dans deux directions différentes: certains constructeurs l'emploient pour ses seules qualités techniques en le cachant derrière des façades classiques, tandis que d'autres utilisent en plus les possibilités nouvelles qu'offre ce matériau du point de vue des formes architecturales. Parmi ces derniers, rares sont ceux qui ne seront pas tentés de décorer leurs structures métalliques avec des motifs qui leur sont étrangers.

6 Eclipsé, après 1918, par un autre matériau, le béton, plus économique, le métal sera presque totalement absent du mouvement de renouveau du début $\mathrm{du} \mathrm{xx}^{\mathrm{e}}$ siècle, durant lequel furent jetées les bases de l'architecture ocntemporaine. Il faudra attendre les années 1930 pour que l'architecture métallique réapparaisse en France, en s'appropriant les éléments de la nouvelle pensée architecturale - refus du décor, renouveau des formes et des structures - et retrouve ainsi un nouveau souffle.

7 Dès lors, le métal s'introduira dans tous les domaines de la construction contemporaine et, sous des formes structurelles spécifiques et très diverses, il permettra aux architectes et aux ingénieurs qui l'utilisent de créer des espaces nouveaux propres au temps dans lequel ils s'inscrivent.

8 Ainsi, expression particulière du va-et-vient permanent entre un passé toujours vivant et un esprit nouveau qui cherche à s'imposer, l'histoire de l'architecture métallique de notre pays met en évidence deux phénomènes essentiels.

\section{Le métal et l'architecture française}

9 Le premier phénomène réside dans les progrès très importants que l'architecture métallique a fait faire au processus d'industrialisation du cadre bâti. Le secteur du bâtiment reste certes, encore de nos jours, très archaïque dans ses procédés et l'on peut considérer que, de ce point de vue, l'architecture française en est encore à sa préhistoire - contrairement à d'autres secteurs d'activités comme celui de l'automobile, par exemple, où l'on n'hésite pas à engager des sommes considérables en études pour mettre au point des systèmes qui permettent in fine de produire à moindre coût des produits industriels de grande qualité. Au stade actuel, le choix offert par les composants du marché est encore réduit et les concepteurs doivent, soit créer eux-mêmes des systèmes qui peuvent être industrialisés, soit s'adapter à ceux qui existent et qui sont plus ou moins complets. Il reste que la construction métallique, grâce aux apports successifs des architectes et des ingénieurs depuis plus d'un siècle, connaît aujourd'hui un stade de production 
industrielle en série qui est à l'origine de progrès qualitatifs importants parmi lesquels il faut retenir notamment :

- la rapidité d'exécution des chantiers par le report en atelier d'une partie importante des procédures de fabrication.

- la précision dimensionnelle des structures qui facilite grandement leur mise en oeuvre et favorise l'accueil de tous les éléments de second oeuvre qu'elles supportent et qui peuvent, à leur tour, être préfabriqués avec la même précision.

- la pérennité des ouvrages qui peut être obtenue grâce aux diverses méthodes existant actuellement de protection des métaux contre la corrosion ou contre l'incendie, qui permettent d'assurer une durée de vie très longue aux ouvrages métalliques. Cette pérennité réside également dans les possibilités qu'offrent les structures montées à partir d'éléments industrialisés de remplacer un ou plusieurs de ces éléments, voire d'en ajouter.

- la qualité architecturale des édifices construits en métal, cette qualité étant pour partie liée à la diversité évoquée plus haut, mais étant également liée à l'évolutivité, à la flexibilité et à la possibilité de participation des usagers que permet l'emploi du métal par le biais de l'industrialisation ouverte. Ces notions reposent sur l'idée qu'un bâtiment doit pouvoir subir facilement, sans modification de son enveloppe générale, des transformations, non seulement durant sa conception et son édification - ce qui est utile au maître d'ouvrage, à l'architecte et ce qui autorise l'intervention de l'usager - mais aussi durant son usage, ce qui peut permettre des adaptations postérieures à sa réalisation.

Le second phénomène qu'il convient de souligner est le rôle fondamental de l'architecture métallique dans tous les débats qui ont trait aux rapports entre l'art et la technique en général, entre l'architecte et l'ingénieur en particulier. L'évolution de l'architecture française en métal a été accompagnée en effet de nombreuses prises de positions que l'on peut, par commodité, classer en trois catégories :

1. Celle qui réduit l'architecture à un art plastique, la fonction n'étant qu'un simple prétexte et la construction le moyen de réaliser un « bel ouvrage ». Cette attitude est celle des traditionalistes.

2. Celle qui pose comme principe que la forme d'un édifice ne peut résulter que de sa fonction. C'est le principe posé par Viollet-le-Duc et souvent repris depuis.

3. Celle qui donne la primauté à la construction. Elle se subdivise en trois attitudes :

- la première consiste à essayer de réunir les «lois éternelles » de l'architecture et de la construction;

- la seconde postule que la technique met fin aux architectures antérieures;

- la troisième met en avant l'exigence de réalisation de structures non spécifiques dans lesquelles peuvent s'insérer des espaces fonctionnels.

11 Il faut convenir que ces débats se poursuivent sans renouvellement réel depuis le XIX siècle. Nous avons pu le constater récemment avec ceux qu'a engendré la réalisation dans Paris de plusieurs édifices marquant son site. Ces prises de positions sont étonnamment similaires à celles qu'avaient pu provoquer des édifices construits il y a cent ans, en particulier la Tour Eiffel.

Le métal a également une influence non négligeable sur l'évolution des rapports entre architectes et ingénieurs (qui recouvre en partie l'évolution des idées évoquées plus haut), car la maîtrise de ce matériau n'a jamais pu être assurée en totalité, ni par les architectes seuls qui n'en ont pas - tout au moins en France - la capacité technique, ni par les ingénieurs seuls que leur formation n'a pas préparé à des activités de création purement architecturales. Ainsi, séparées à leur début par des conceptions radicalement 
opposés, les deux professions ont dû, depuis quelque temps, amorcer un rapprochement certain. A ceci s'ajoute le fait, plus général, que les architectes considèrent de plus en plus fréquemment, depuis le début $\mathrm{du} \mathrm{xx}^{\mathrm{e}}$ siècle, que leur rôle n'est pas seulement de dessiner une architecture de façade, mais qu'il consiste également, à partir de sa spécificité propre, à satisfaire les besoins sociaux en espaces bâtis en prenant en compte tous les paramètres nécessaires. Cela renforce la nécessité pour l'architecte de s'entourer de spécialistes dans tous les domaines qui ne sont pas de sa compétence. De leur côté, les ingénieurs se posent de moins en moins en concurrents des architectes et nombre d'entre eux deviennent également favorables à une collaboration accrue. Cherchant en définitive l'union de l'esprit créatif et de l'esprit analytique, architectes et ingénieurs ne sont plus « ennemis » sur le champ de la répartition de la commande ; ils deviennent partenaires.

\section{Les constructions publiques en métal}

limitée, dans un premier temps, aux constructions publiques de l'Etat et des collectivités territoriales dans la Région Ile-de-France. Celle-ci se situe dans le prolongement de la thèse "Métal et architecture dans la France moderne et contemporaine" qu'a récemment soutenue Frédéric Seitz (thèse dirigée par Marcel Roncayolo). En raison de l'absence de documents de travail évoquée plus haut, cette thèse a dû être complétée en annexe par:

- un répertoire de 350 constructions françaises réalisées en métal durant les $\mathrm{XIX}^{\mathrm{e}}$ et $\mathrm{XX}^{\mathrm{e}}$ siècles ;

- une série de brèves notices biographiques d'une centaine d'auteurs (architectes ou ingénieurs) de ces réalisations ; ces annexes, succinctes par nature, sont la base du travail de recherche entrepris par le Centre de Recherches Historiques, qui sera mené par le biais des fonds d'archives de l'architecture et de deux enquêtes parallèles :

- l'une auprès des collectivités locales, des Directions Départementale de l'Equipement (D.D.E.) et des Services Départementaux de l'Architecture (S.D.A.) de Paris et de la Région Ile-de-France, pour localiser les constructions publiques réalisées en métal durant la période qui nous intéresse (les $\mathrm{XIX}^{\mathrm{e}}$ et $\mathrm{XX}^{\mathrm{e}}$ siècles) sur les territoires de ces collectivités et pour connaître les noms de leurs auteurs.

- l'autre auprès des architectes et ingénieurs déjà connus pour avoir construit une ou plusieurs oeuvres en métal, pour essayer de déterminer et de comprendre les raisons objectives qui les ont conduits à utiliser ce matériau.

15 Ces enquêtes sont en cours et leur dépouillement devrait être un premier pas vers la rédaction d'un ouvrage de références en deux parties :

- une première partie sera constituée d'une liste aussi exhaustive que possible des constructions publiques réalisées en métal durant la période et dans la région géographique retenues, cette liste devant être complétée par une série de fiches regroupant, pour chaque bâtiment, toutes les informations qui auront pu être recueillies sur :

- sa description, 
- son mode et sa technique de construction,

- les conditions dans lesquelles la décision de la réaliser a été prise,

- les conditions dans lesquelles le métal a été choisi pour son édification,

- les conditions dans lesquelles sa commande a été confiée à tel architecte ou à tel ingénieur,

- les changements d'affectation successifs dont il a été l'objet et les transformations qu'il a subies du fait de ces changements.

- une seconde partie comportera une suite de notices biographiques approfondies des architectes et ingénieurs auteurs des bâtiments de la partie précédente ; ces biographies auront pour objet d'apporter des précisions sur :

- les milieux sociaux des personnalités étudiées,

- leur formation,

- leur carrière professionnelle,

- les raisons (techniques, financières, idéologiques...) qui les ont conduits à utiliser le métal,

- leurs rapports avec la maîtrise d'ouvrage dans le cadre des opérations étudiées.

L'objectif recherché en réalisant un tel outil est de répondre aux nombreuses questions importantes que posent la maîtrise d'oeuvre et la maîtrise d'ouvrage public. Nous espérons aussi, au travers de cette analyse, faire apparaître les constructions publiques des $\mathrm{XIX}^{\mathrm{e}}$ et $\mathrm{XX}^{\mathrm{e}}$ siècles, réalisées en métal, comme des témoignages d'architecture qu'il convient de traiter de la même façon que les bâtiments construits antérieurement, c'està-dire avec le souci de leur conservation et de la permanence de leur utilisation. Cette recherche devrait donc intéresser non seulement des équipes universitaires, mais aussi des professionnels du cadre bâti (architectes, ingénieurs, entrepreneurs) et des professionnels de l'industrie du métal qui ont déjà, à plusieurs reprises, manifesté leur intérêt pour des études menées sur ce mode de construction. Ce travail devrait donc être l'occasion d'un rapprochement entre la communauté universitaire et l'ensemble de ces professionnels.

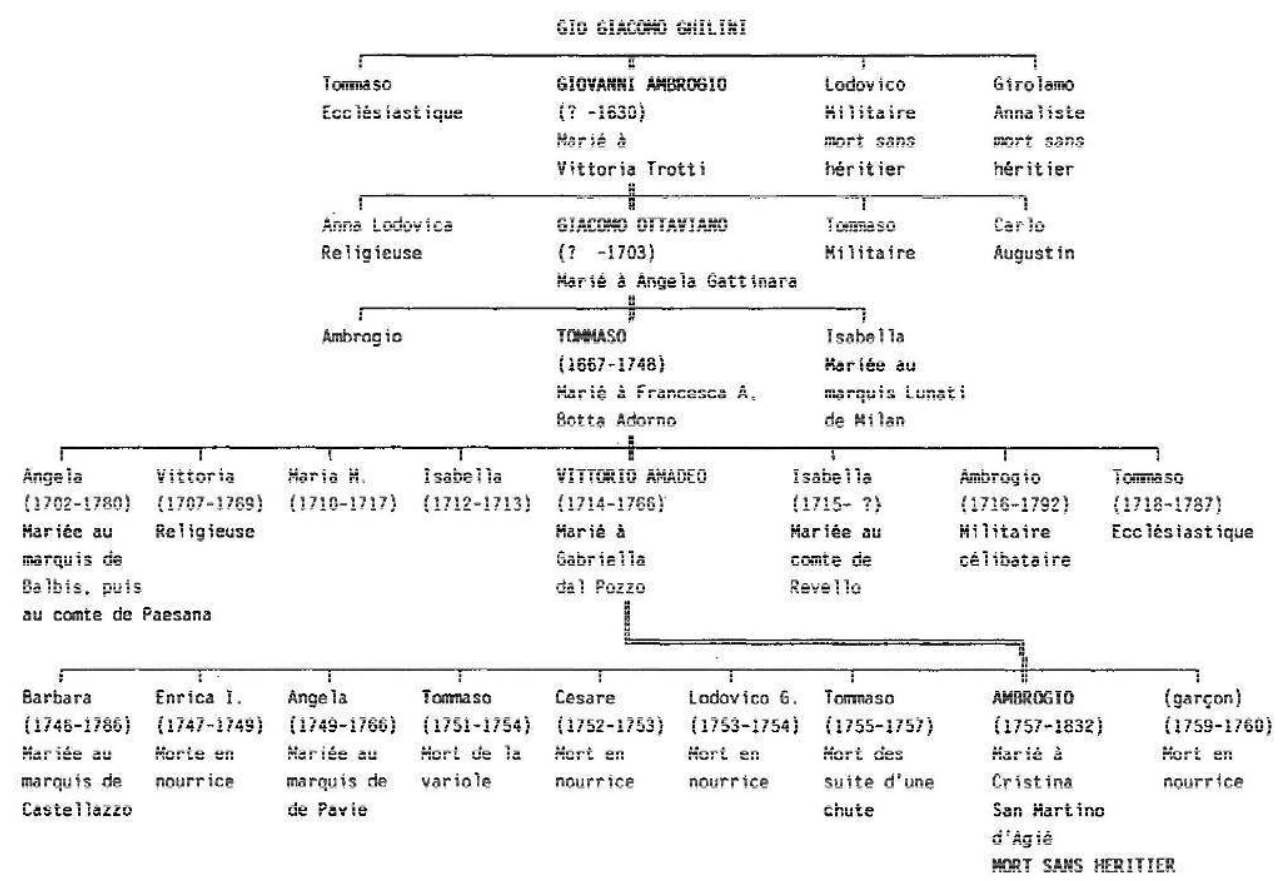

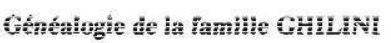

